\title{
Demo Abstract: SmartH2O, demonstrating the impact of gamification technologies for saving water
}

\author{
Andrea Emilio Rizzoli ${ }^{1}\left(\right.$ Andrea Castelletti $^{2}$ - Piero Fraternali ${ }^{2}$. \\ Jasminko Novak ${ }^{3}$
}

(C) Springer-Verlag GmbH Germany 2017

\begin{abstract}
SmartH2O is a software platform that creates a virtuous feedback cycle between water users and the utilities, providing users information on their consumption in quasi real time, and thus enabling water utilities to plan and implement strategies to reduce/reallocate water consumption. The SmartH2O platform adopts a gamification paradigm to motivate users to change their water use behaviour and different incentives (virtual, physical, and social) are used to stimulate the competition among users. The SmartH2O platform, developed during the EU-FP7 project that bears the same name, has been deployed in two test sites: in Terre di Pedemonte, a small municipality in Tessin, Switzerland, and in Valencia, a large urban centre, in Spain. Thanks to its use it has been observed an average reduction in consumption of $10 \%$ in Switzerland and of 20\% in Spain among the platform adopters.
\end{abstract}

Andrea Emilio Rizzoli

andrea.rizzoli@supsi.ch

Andrea Castelletti

andrea.castelletti@polimi.it

Piero Fraternali

piero.fraternali@polimi.it

Jasminko Novak

j.novak@eipcm.org

1 Dalle Molle Institute for Artificial Intelligence (IDSIA), University of Lugano (USI), University of Applied Science and Arts of Southern Switzerland (SUPSI), Galleria 2, Via Cantonale 2c, 6928 Manno, Switzerland

2 Department of Electronics, Information and Bioengineering, Politecnico di Milano, Piazza L. da Vinci 32, 20133 Milan, Italy

3 EIPCM, Pariser Platz 6, 10117 Berlin, Germany
Keywords Water saving · Gamification · Behavioral Change

\section{Stimulating behavioural change for water saving}

Most researchers have so far focussed on promoting behavioural change for energy systems, while water has received less attention, but the increasing diffusion of smart water meters has opened up the way to a number of applications, from user demand profiling [1], to innovative pricing schemes [2], from automated leak detection to providing feedback on water consumption. The opportunities offered by the new metering infrastructure allow water utilities to approach the problem of balancing water demand and supply from a different angle: instead of constantly increasing infrastructures for water supply, one can now try to modulate and restrain demand, especially in peak times.

The European Commission has therefore launched a series of projects with the objective of exploiting the new ICT infrastructure for a more efficient and sustainable water management. These projects have been grouped in the "ICT4Water cluster"1 and the SmartH2O project was one of such projects: still funded in the previous FP7 framework programme it ended in March 2017.

The objective of SmartH2O was to explore how water demand, and therefore the user behaviour, could be influenced by different types of stimuli: physical incentives, awards, increased awareness, social norm. For this purpose, a specific software platform has been realised.

\footnotetext{
${ }^{1}$ http://www.ict4water.eu.
} 


\section{The SmartH2O platform}

In order to verify the scientific assumptions of the project, a software infrastructure has been set up involving a data flow from smart meters to end users. Smart water meter measurements are used for two main purposes: consumption data are displayed to the users through a gamified interface; the same data are used to profile the user consumption patterns and to disaggregate the total water consumption into the single end uses. This latter information is used to determine which stimuli to use to motivate users towards an increased water efficiency.

In Fig. 1 a detail of the gamified user interface is shown. The user is presented with an intuitive view of his/her water consumption and a projection over the whole year. Te user can then decide which saving goal to self impose. A more ambitious goal will bring back more points.

Users can accumulate points by saving water, but also by performing actions on the platform, such as reading water saving tips, or providing data on the household characteristics that are useful for user profiling. Points are displayed in leaderboards as shown in Fig. 2. Once the users have acquired a given number of points they can claim physical rewards, such as the board game "Drop", which was purposely designed for the SmartH2O project. Drop is a hybrid game with a matching digital counterpart which integrates in the gameplay, in order to allow users to get points also when they play the educational game "Drop".

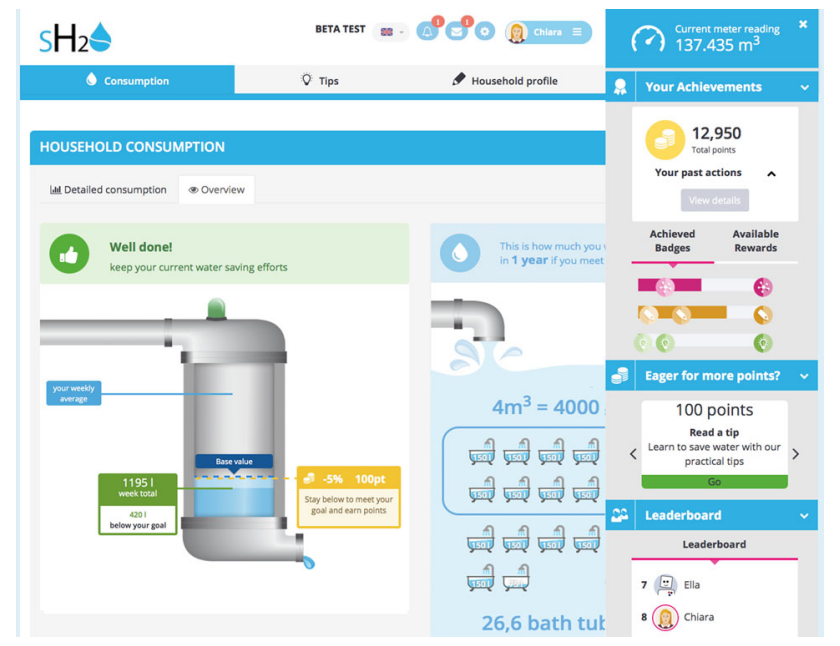

Fig. 1 The goal setting mechanism

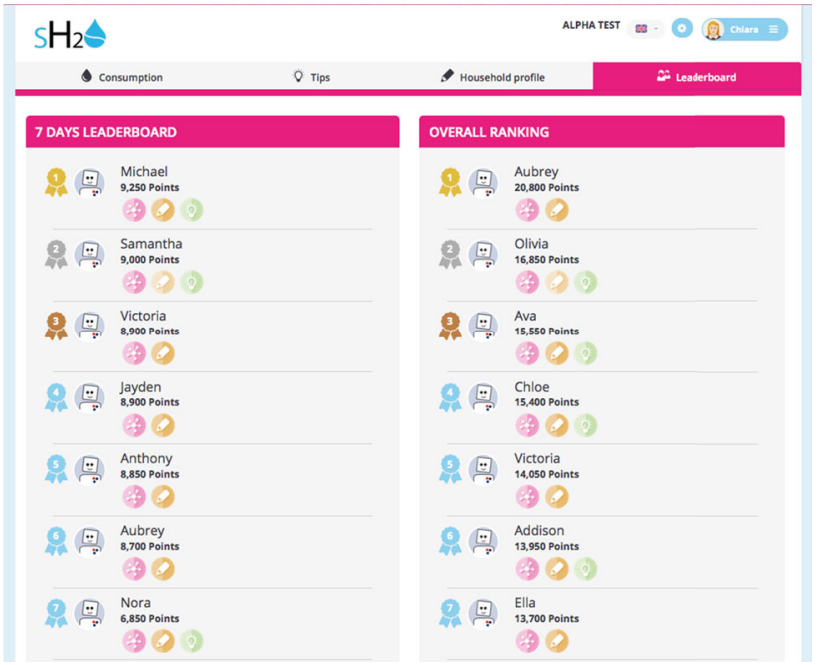

Fig. 2 The weekly and the overall leaderboards

\section{Conclusions}

SmartH2O is a FP7 project which ran from 2014 until 2017. In this time span it was successfully deployed in two real world case studies, and in the Valencia case it was fully integrated in the business logic of the Virtual Office of Global Omnium, the water utility of Valencia, which serves more than 400'000 households, thus proving its readiness for industrial-level deployments. SmartH2O users have consistently saved water in comparison to non-users, with users consuming $20 \%$ less water than non-users.

Acknowledgements This work is partially supported by the SmartH2O project funded by the EU FP7 Programme, Grant Agreement No. 619172 .

\section{References}

1. Cominola A, Giuliani M, Piga D, Castelletti A, Rizzoli A (2015) Benefits and challenges of using smart meters for advancing residential water demand modeling and management: a review. Environ Model Softw 72:198-214. doi:10.1016/j.envsoft.2015.07.012

2. Harou J, Garrone P, Rizzoli A, Maziotis A, Castelletti A, Fraternali P, Novak J, Wissmann-Alves R, Ceschi P (2014) Smart metering, water pricing and social media to stimulate residential water efficiency: opportunities for the SmartH2O project. Procedia Eng 89:10371043. doi:10.1016/j.proeng.2014.11.222 